\title{
OPTICAL WAVEGUIDING SYSTEMS: CONSTRUCTIONS AND APPLICATIONS
}

\author{
Karush Suri $^{1}$, Vishakh Mehta ${ }^{2}$ \\ ${ }^{I}$ Student, Department of Electronics and Communication Engineering, Amity University, Uttar Pradesh, India \\ ${ }^{2}$ Student, Department of Mechanical Engineering, JSS Academy of Technical Education, Uttar Pradesh, India
}

\begin{abstract}
The basic geometry of a waveguide reflects its functions. These consist of wave simulation for information carriers, guiding and networking in the telecommunication industry, applying the laser guided modes in various research areas such as particle dispersion and randomization effect theories. The basic uitlity of a waveguiding system is determined by the optical efficiency of that system, which is the percentage ratio of effective transmission carried out by that particular waveguide. Advanced guiding networks consist of complex tubes with different alterations in their sizes in order to fulfill this requirement. As the industry grows, so does the demand for wave simulation techniques and thus, day by day new methods are being developed which focus on the development of effective wave propogation. Another important factor which is included in the designing of wave guiding devices is the frequency of the optical signal. A signal with significantly higher frequency bands will be guided through a complex system which in turn does not guide waves of lower frequency. Conventional designs have been consisting of the long hollow metallic tubes which guide the electromagnetic signals towards the receiving ends. However, slight deviation is being observed in the newly enhanced devicing.
\end{abstract}

Keywords- Cladding, Spatial Accumulation, Photorefractive, Wave-Coupling

\section{INTRODUCTION}

Modern waveguiding systems find their applications in a number of scenarios dealing with laser photonics and optics of complex networks. Suspension of particles is one of the most important domain consisting of waveguiding methods which determine the proper integration of photonic particles as information carriers. A number of these systems can be deviced for single mode propogation as well as for reducing optical losses in the telecommunication field. One of the most important factor required in carrying out the wave simulation process is the total internal reflection. Especially in case of dielectric waveguiding ${ }^{[1]}$ where a number of layers are involved, only a discrete set of angles are used for the slab which are in approximation to the critical angle. Attenuation upto some extent might be witnessed in a number of experiments where the waveguiding regarding photonic species is associated.

\section{THE CONVENTIONAL DESIGNING}

Traditional devicing consists of long hollow metallic tubes, rectangular edged boundaries and some basic knowledge of propogating mechanics. However, these do not solve the complete purpose of effective transmission. A number of losses are associated with these systems. These may have a significant impact on the state and orientaion of the signal. For example, high frquency signals might have thier information carrying capacity reduced if a proper guide (consisting of tough exterior coating) is not used. This will result in data loss and thus, harm the overall transmission process.

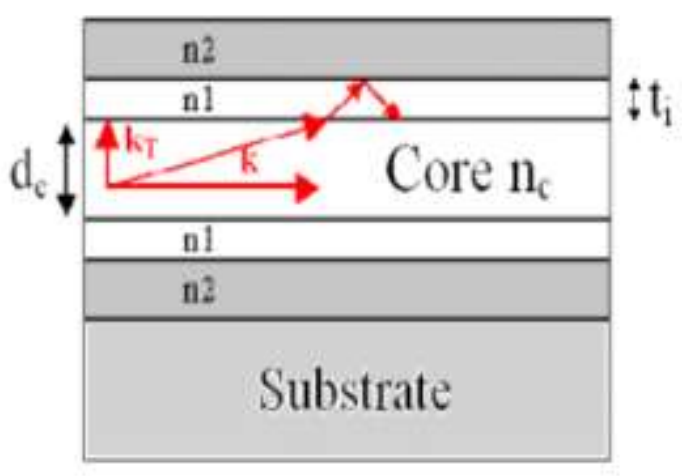

The figure above depicts the basic functioning of a simple waveguiding system. Light enters through one of the open ends of the tube and undergoes a series of total internal reflections(due to the signficant differences in the refractive indices) after which it is delivered to the receiver. The complete signal is transmitted and all its qualties are preserved as a result of this reflection array. Cladding is the outer coating of the device which protects the interior core from exterior mechanical disturbances. An effective simulation system consists of a tough cladding which prevents signal losses.

Commonly, strip waveguides ${ }^{[2]}$ are rectangular waveguides with a strip of the layer confined between multiple cladding layer. These are the most commonly used form of waveguiding networks and consist singular transverse directions. These have a number of application scenarios starting from integrated optical circuits to wavelength 
divsion multipliers which deal with the division of various components of a particular wavelength at the nano level.

A number of waveguides allow periodic alterations in their sizes and dimensions. This does not accomodate on the loss of the signal. These have varying cross sections and are differently utilized in the industrial domain. Such waveguides are coined as segmented waveguides and deal with the transmission in terms of only certain segments which are involved in their propogation.

\section{ADVANCED DEVICING AND}

\section{CONSTRUCTION METHODS}

\subsection{Laser Inscribed Waveguides}

Configuration of optical waveguides in proper orientation gives rise to the proper designing of electronic components in optical circuits and complex chip networks. Usually, such waveguides are deisgned for serving the purpose in telecommunication relay applications where a number of wavelengths are involved.

These are constructed with the help of photorefractive effect in near transparent materials. Non linear absorption ${ }^{[3]}$ of pulse laser is observed which causes a decreament in he refractive index. In order to eliminate this problem of refractive index, laser pulses of very short duration (commonly femtosecond) are used which increase the value refractive index. With the help of translation of the focal spot through a bulk transparent material, the waveguides can be easily simplified and written. Slight variations in this methods might be observed. These include the use of a low NA microscope objective and translation of the beam along the beam axis. This causes a significant improvement in the overlap between focused laser beam and the photorefractive material.

Exposure of the transparent material to an unfocused laser beam of higher brightness to initiate photorefractive effect leads to self-focusing. This means that the waveguides form themselves on thier own due to spatial accumulation. Such formation of waveguides leads to the discontinuity of the laser.

\subsection{Waveguides in Practice}

Practically, waveguiding systems are the building blocks of super high frequency systems. These are the main channels which transmit complex signals. Rectangular waveguides are used to design waveguides in which the frequency band ratio is significantly high. Waveguide dimensions should be carefully noted and kept in mind while developing the design as they play a key role in the porcess.

Due to the large bandwidth propogation property of a single mode laser in cases of rectangular guides, a number of standards exist for these waveguides. These are noted while designing the waveguide and are followed in strict accordance in order to reduce transmission losses.

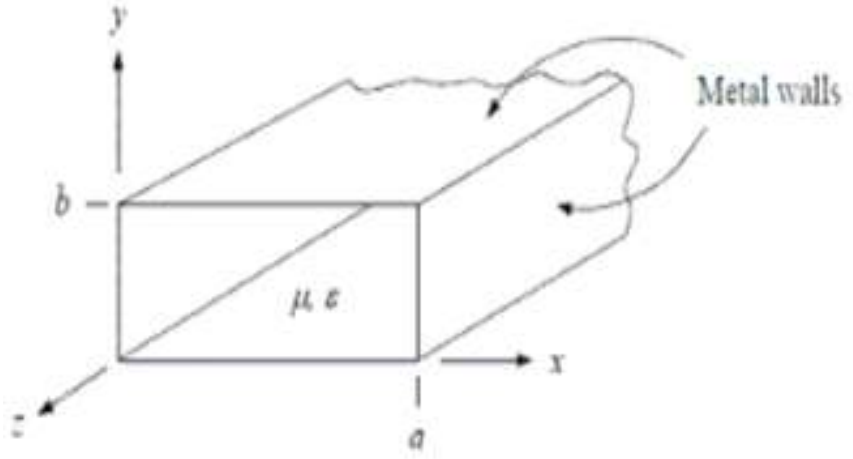

Another issue which arises during the propogation of wave signals is the elimination of losses. Clearly, the process is a bit tedious and involves some loss of data which cannot be neglected. A number of internal processes help in reducing the overall loss. These include dispersion and evanescent wave-coupling ${ }^{[4]}$. Dispersion is a phenomenon in which the velocity of propogation of the signal is denoted as a function of its frequency. It is one of the many important factors as it reduces the loss per unit length. Similarly, evanescent wavecoupling refers to the coupling between two waves due to the physical overlapping. The evanescent field generated by a wave excites the other wave in the fiber. Losses can be significantly reduced if the excitation of these wave particles is controlled. This would result in non-simulated propogation and less loss of information.

\section{WAVEGUIDE FABRICATION}

A number of fabrication techniques are being used to design new and modified types of waveguiding systems. Commonly used method is that of the deposited thin films. Millions of inexpensive waveguides are needed to fully implement the networking process across the globe, also known as the "information superhighway" ${ }^{[5]}$. The process of deposition mainly includes a number of complex methods such as spinning or dipping, as well as vacuum vapor deposition and sputtering.

Thermally stimulated vacuum- vaporation is the standard method for producing thin films of conventional design. However, the amount of loss in this amnner is comparitively high $(10 \mathrm{Db} / \mathrm{cm})$. This high loss is a result of the inclusion of contaminant atoms that act as absorption and scattering centers.

Instead, sputtering deposition of molecules is used from a solid source. Sputtering consists of the atoms or molecules which are removed from the surface of a source (target) material, in vacuo, by the subsequent bombardment with ions having energies in excess of about $30 \mathrm{eV}$ to about 2 $\mathrm{KeV}$. The atoms removed from the surface of the source are allowed to deposit on the surface of a substrate, forming a thin layer. The sputtered thin film is built slowly by the accumulation of individual particles arriving at the surface with significantly higher kinetic energies. The result of this complex process is a fine unform layer due to the uniform kinetic distribution of the particles on the surface. 


\section{CONCLUSION}

Waveguiding systems find their applications in a number of modern day scenarios. These include data relaying, algorithm processing and development, data simulation and analysis of networking instruments used in a number of transmission processes. Construction and organization of these devices follows a definite method. Each waveguide can be designed as per its specific functions and then used to carry out the basics of transmission. Research opportunities in this sector include a number of modified methods which aim at reducing the losses due to propogation and due to the rigid body structure of these waveguides. Later part of the paper focusses on two of the most important fabrication techniques. These consist of various intrinsic mechanical processing such as dripping and sputtering. Simplified procedures include assessment and analysis of the design and then obtaining data friendly optical guiding systems. Main focus is always on minimising data losses and and making the simulation process effective and efficient.

\section{REFERENCES}

[1]. Marcatili, E. A. J. (1969). "Dielectric rectangular waveguide and directional coupler for integrated optics". Bell Syst. Tech. J. 48: 2071-2102.

[2]. Kumar, A., K. Thyagarajan and A. K. Ghatak. (1983). "Analysis of rectangular-core dielectric waveguides-An accurate perturbation approach". Opt. Lett. (8): 63-65.

[3]. Kerry J. Vahala, Nature 424, 839-846 (14 August 2003),doi:10.1038/nature01939

[4]. R. Behrisch (ed.): Sputtering by Particle Bombardment I, Topics Appl. Phys., Vol.47 (Springer, Berlin, Heidelberg 1981)

[5]. B.L. Booth: Optical interconnection polymers, in Polymers for Lightwave and Integrated Optics: Techniques and Applications, ed. by L.A. Hornak (Dekker, New York 1992) p. 232

\section{BIOGRAPHIES}

Born on 17th March, 1997, Mr. Karush completed his education from Amity International School, Noida and shows a keen interest in the field of communication networking. He has published a number of papers in many prestigious journals such as the International Journal of Electronics and Communication Engineering and the International Journal of Science and Research. He is currently pursuing his Bachelor of Technology in the branch of Electronics and Communication Engineering from Amity University, Uttar Pradesh.

Born on 31st August, 1997, Mr. Vishakh Mehta completed his education from and shows a keen interest in the field of Mechanical Development and Automation Designing. He has worked on a number of research projects and achieved success in most of them. Some of these include newer and effective methods of developing Optical Waveguides. Vishakh is currently pursuing his Bachelor of Technology in the branch of Mechanical Engineering from JSS Academy of Technical Education, Uttar Pradesh. 